\title{
دراسة اقتصادية للإستثمارات الأجنبية في بعض دول الخليج
}

\author{
يعقوب عحمد أحمد محمود محمد القطان \\ طالب دكتوراه بمعهد البحوث والدراسات الأفريقية - جامعة أسوان
}

عحم عبد الحفيظ محمد

استاذ الاقتصاد الزراعي وعميد كلية الزراعة - جامعة سوهاج

حسن موسي رضوان

مدرس الاقتصاد الزراعي بالمعهد العالي للتعاون والإرشاد الزراعي بأسيوط 
الملخص:

تهاف الدراسة الي التعرف علي الوضع الحالي لتدفقات الاستثمارات الأجنبية المباشرة في كل من السعودية والامارات والكويت سواءالبينية أو الواردة من العالم. حصلت المملكة العربية السعودية من تدفقات الاستثمارات الأجنبية المباشرة علي حوالي 19 مليون دولار كمتوسط فترة الدراسة، تمثل حوالي Vr\% من حجم الاستثمارات الأجنبية المباشرة الواردة الي دول الخليج العربية، بينما حصلت الامارات من تدفقات الاستثمارات الأجنبية المباشرة علي حوالي 9.00 مليون دولار كمتوسط فترة الدراسة، تمثل حوالي سء\% من حجم الاستثمارات الأجنبية المباشرة الواردة الي دول الخليج العربية، بينما حصلت دولة الكويت من تدفقات الاستثمارات الأجنبية المباشرة علي حوالي §. أ مليون دولار كمتوسط فترة الدراسة، تمثل حوالي ج\% من حجم الاستثمارات الأجنبية المباشرة الواردة الي دول الخليج العربية.

الكلمات الإفتتاحية: الإستثمارات الأجنبية - دول الخليج العربى - الاخل القومى

\section{Abstract}

\section{An Economic Study of Foreign Investments in the Gulf States}

The study aims to identify the current situation of foreign direct investment flows in Saudi Arabia, the UAE and Kuwait, whether intra-regional or imported from the world. From the inflows of foreign direct investment, the Kingdom of Saudi Arabia got about \$ 8.19 million as the average for the study period, representing about $37 \%$ of the volume of foreign direct investment coming into the Arab Gulf states, while the UAE got from the inflows of foreign direct investment about $\$ 9.55$ million as the average of the study period, It represents about $43 \%$ of the volume of foreign direct investments coming into the Arab 
Gulf states, while the State of Kuwait obtained from the inflows of foreign direct investments about $\$ 1.24$ million as the average period of study, representing about $6 \%$ of the volume of foreign direct investments coming into the Arab Gulf states.

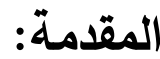

شهد العالم اثثاء العقود الماضية زيادة هائلة في تدفقات رؤوس الاموال العالمية، وتعزي هذه لزيادة في حجم التدفقات الاستثمارية في جزء كبير منها الي حقيقة مفادها

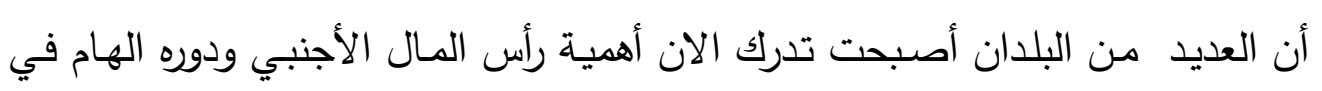

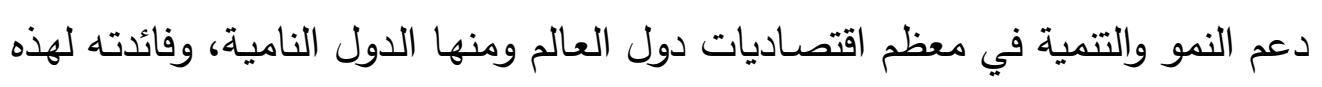

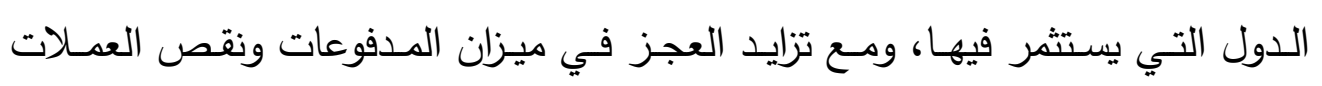

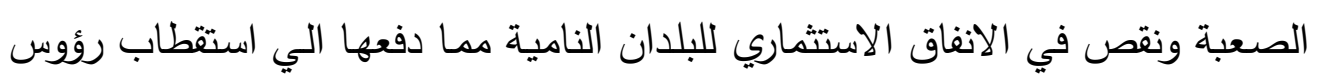

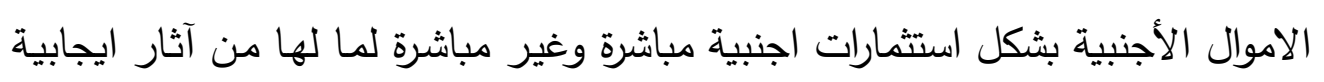

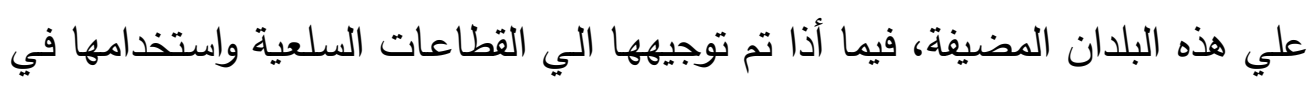

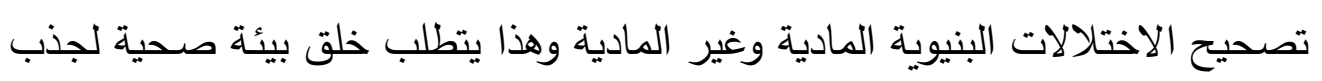

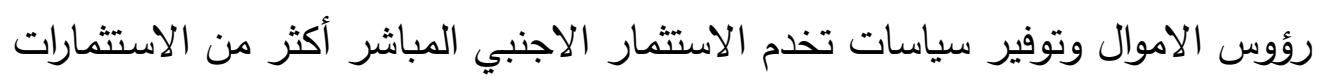

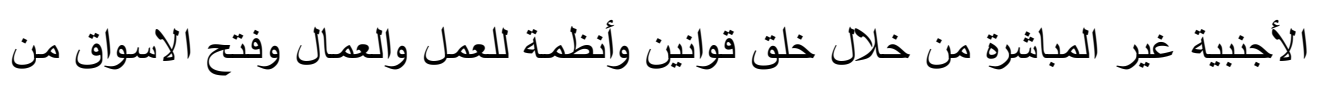
خلال تقديم حوافز خصومات ضريبية، وحماية حقوق الملكية الفكرية والمادية فضـلا

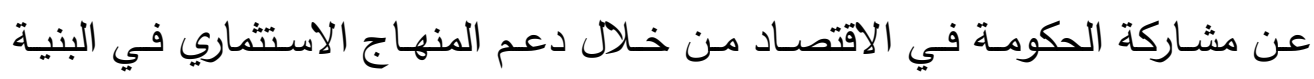

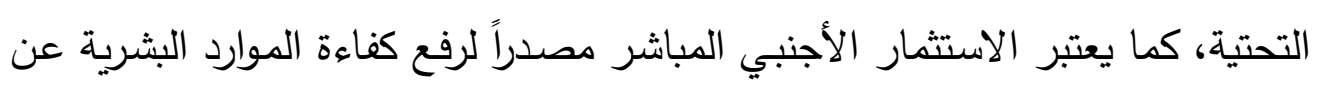
طريق مساهمته الفعالة في نقل المعرفة وتعليم وتدريب هذه الموارد. بالإضافة إلى دوره

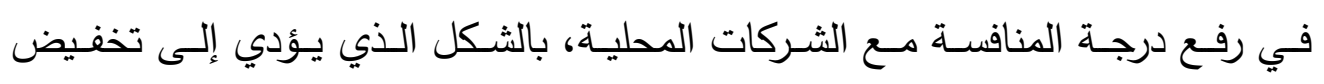

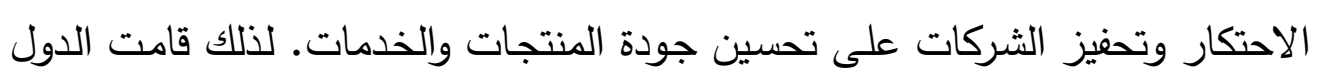


النامية بحاولة خلق مناخ ملائم لاستقطاب الاستثمارات الأجنبية المباشرة من خلال التثريعات المحفزة والمغرية ومحاولة إزالة كل العراقيل.

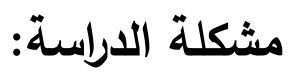

علي الرغم من الجهود التي تبذلها دول الخليج العربية لتحسين مناخ الاستثمار

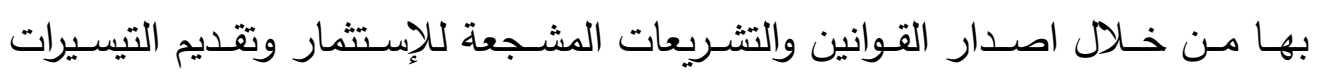

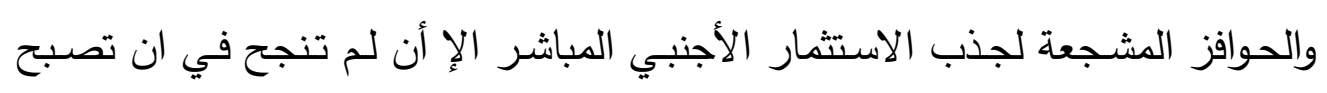
مواقع جذب مهمة للاستثمار الأجنبي المباشر مقارنة بغيرها من الدول النامية الأخري.

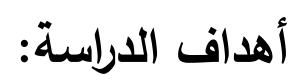

التعرف علي الوضع الحالي لتدفقات الاستثمارات الأجنبية المباشرة في كل من

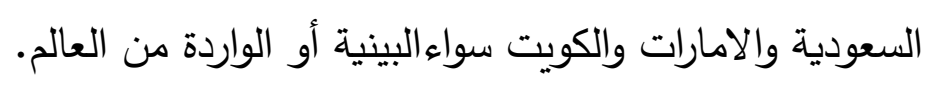

نطاق الاراسة: تُطبق هذه الدراسـة في المملكة العربية السعودية والامـارات والكويت وتدفقات

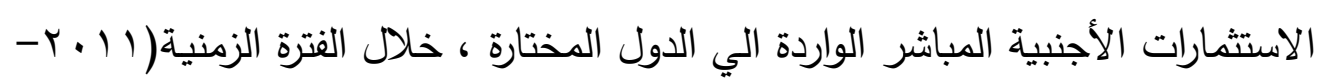

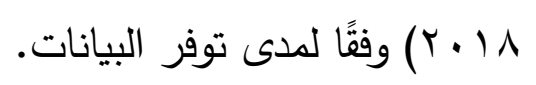

وهناك عدة تعاريف للاستثمارات الأجنبية المباشرة نورد أهمها في ما يلي:

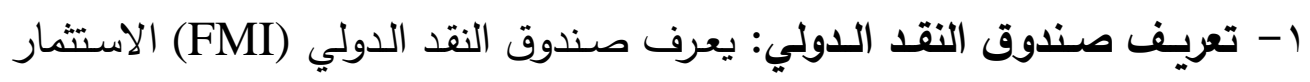

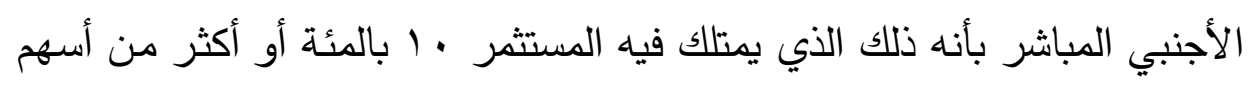

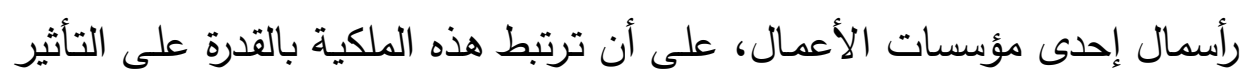

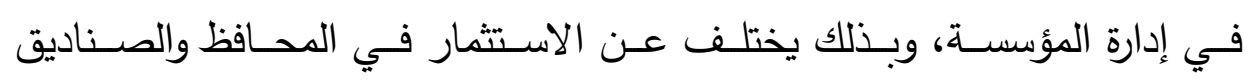

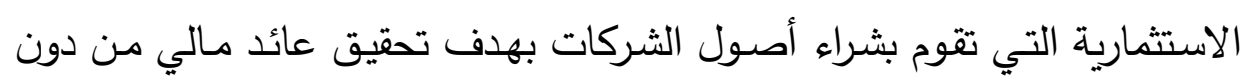


التحكم في إدارتها، مـع ضـرورة التتبيه إلى صـوبة التفريق بثكل دقيق بين النوعين.

ץ- تعريـف منظمـة التعـاون والتنميـة الاقتصـادية: تعرف منظمـة التعاون والتتميـة

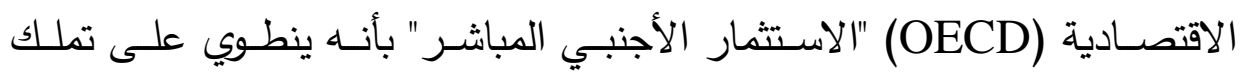

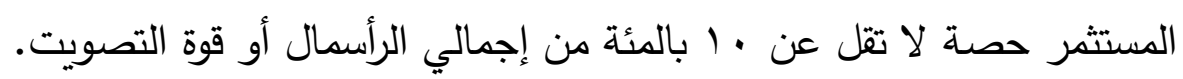

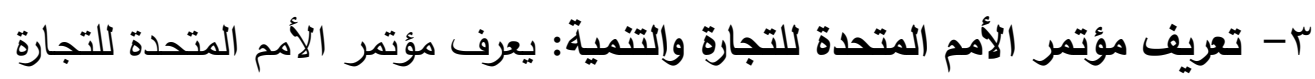

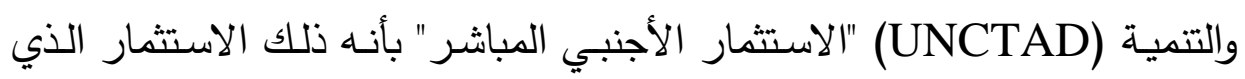

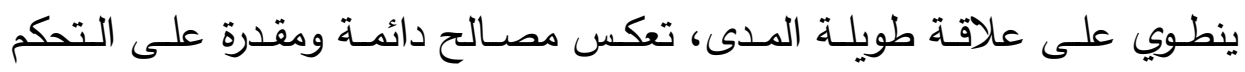

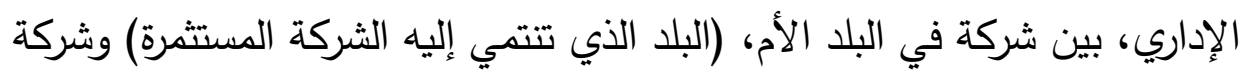
أو وحدة إنتاجية في بلد آخر (البلد المستقبل للاستثمار) .

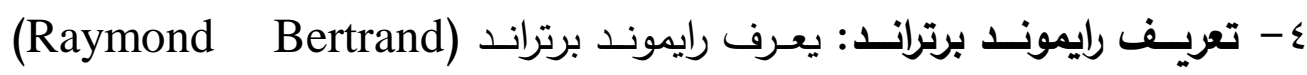

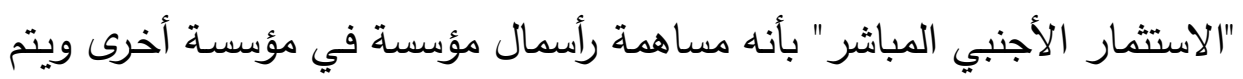

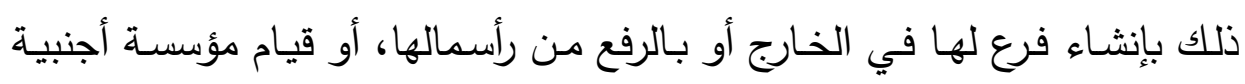

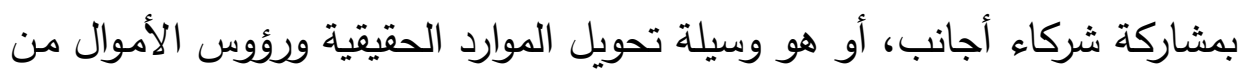
دولة إلى أخرى وخصوصاً عند إنثاء المؤسسة. نتائج الدراسة: المبحث الأول: الاتجاهات الجغرافية والقطاعية للاستثمار الأجنبي المباشر:

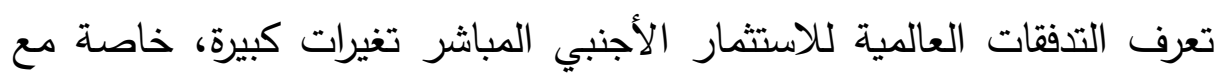
تحول الكثير من اقتصاديات الدول من اقتصاديات الدول مخططة الي اقتصاديات

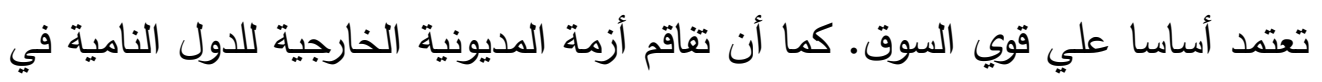

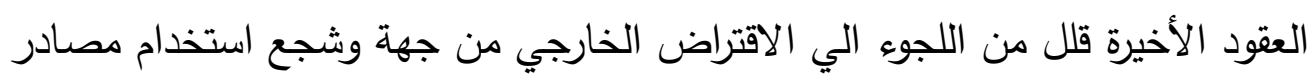
التمويل الخارجية الأخري، ومنها الاستثمار الأجنبي بنوعية المباشر وغير المباشر. 
المطلب الأول: تطور التدفقات العالمية للاستثمار الأجنبي المباشر خلال الفترة

$$
(r \cdot \mid \Lambda-r \cdot 11)
$$

اولأ: الوضع الحالي للأستثمار الأجنبي المباشر الوارد بمناطق العالم: تثير بيانات الجدول رقم (1) والثكل رقم (1) الي حجم الاستثمارات الأجنبية

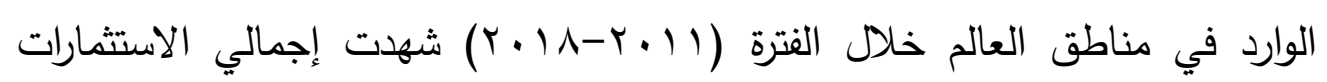
الأجنبية المباشرة الوارد الي مناطق العالم المختلفة تذبذبا واضحا خلال فترة الدراسة

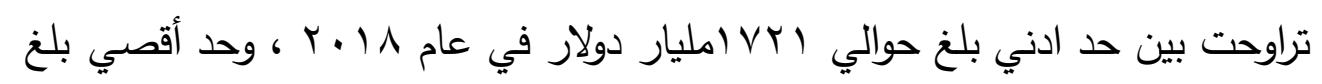

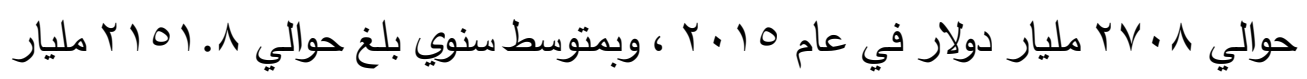
دولار • أما حجم الاستثمارات الأجنبية الوارد الي الدول المتقدمة تراوحت بين حد ادني

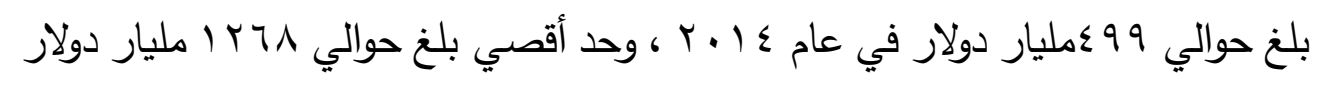

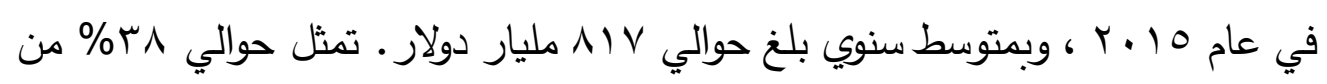
جملة تلك الاستثمارات. أما حجم الاستثمارات الأجنبية الوارد الي الدول النامية تراوحت

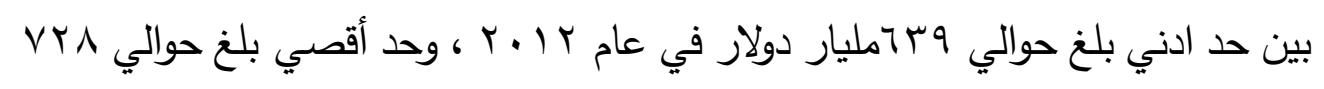
مليار دولار في عام 10 • r ، وبمتوسط سنوي بلغ حوالي TAV مليار دولار ـ تمثل حوالي rr\% من جملة تلك الاستثمارات، أما حجم الاستثمارات الأجنبية الوارد الي افريقيا

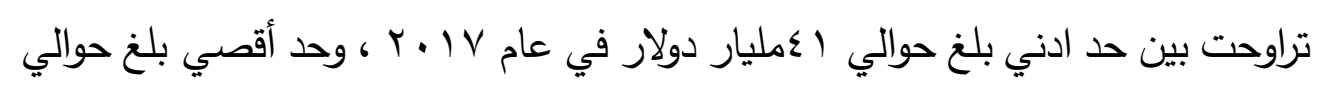

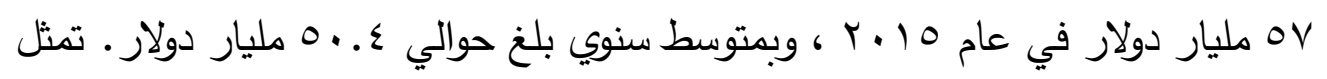
حوالي r\% من جملة تلك الاستثمارات، أما حجم الاستثمارات الأجنبية الوارد الي أمريكا اللاتينية تراوحت بين حد ادني بلغ حوالي هب امليار دولار في عام 7 أ ب ، وحد

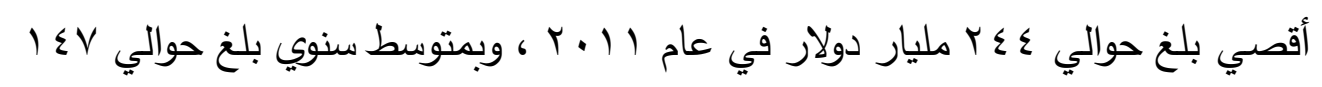
مليار دولار. تمثل حوالي ^^\% من جملة تلك الاستثمارات، أما حجم الاستثمارات

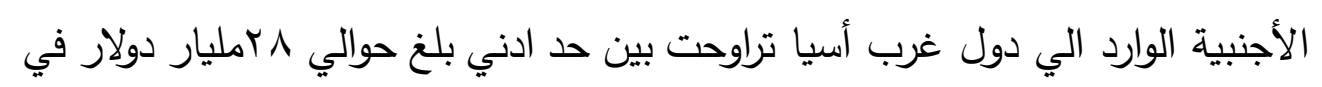




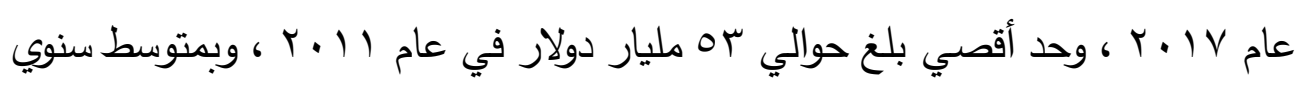

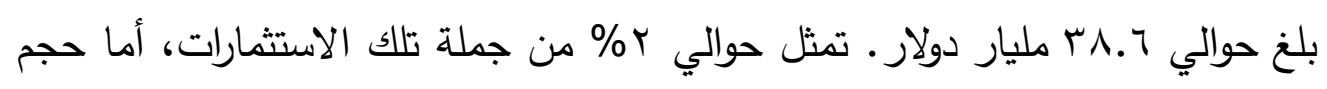
الاستثمارات الأجنبية الوارد الي دول شرق وجنوب أسيا تراوحت بين حد ادني بلغ حوالي

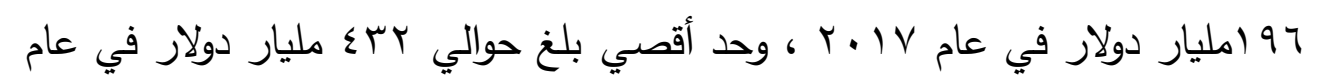

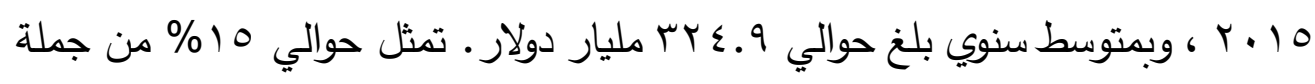
تلك الاستثمارات، أما حجم الاستثمارات الأجنبية الوارد الي الدول المتحولة تراوحت بين

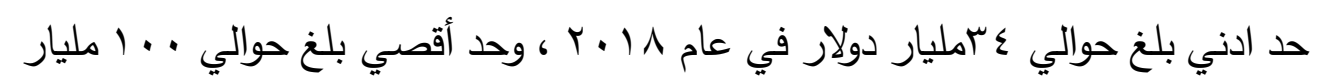

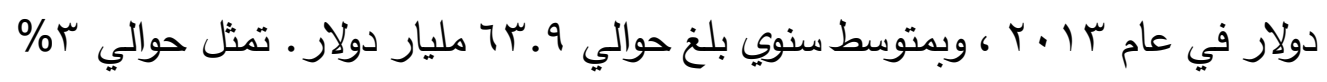
من جملة تلك الاستثمارات.

جدول رقم (1) تظور التدفقات العالمية للاستثمار الاجنبي المباشر الوارد خلال الفترة

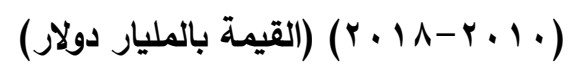

\begin{tabular}{|c|c|c|c|c|c|c|c|c|}
\hline الاجمالي & الدول & جنوب أسيا & غرب أسيا & أمريكا & افريقيا & الألامية & الدمتقدمة & السنوات \\
\hline rTVA & 90 & سזr & or & $r \leq \varepsilon$ & $\varepsilon \wedge$ & VYO & $\wedge \wedge$. & $r .11$ \\
\hline$r \ldots T$ & 10 & ו & $\leqslant \wedge$ & IVA & 07 & q & $7 \vee q$ & $r .1 T$ \\
\hline rI. & $1 \ldots$ & $r \leq \Lambda$ & $\leq 0$ & 117 & $0 \leqslant$ & $7 V 1$ & $79 V$ & r. Tr \\
\hline 1170 & $\leqslant \wedge$ & "ᄉl & $\varepsilon r$ & 109 & $0 \leqslant$ & 711 & $\leqslant 99$ & $r \cdot 1 \varepsilon$ \\
\hline$r V \cdot A$ & דr & $\varepsilon r$ & ו & 107 & or & VYA & $1 Y 71$ & $r .10$ \\
\hline rol1 & 70 & ऍ人т & r & ס & $\leq V$ & 707 & $119 V$ & $r .17$ \\
\hline 1918 & $\leqslant \wedge$ & 197 & rA & 100 & $\varepsilon 1$ & 79. & voq & $r .1 \mathrm{r}$ \\
\hline$|V Y|$ & $r \varepsilon$ & $r \cdot r$ & rq & $1 \leqslant V$ & $\leqslant 7$ & $V \cdot T$ & $00 \mathrm{~V}$ & $r .11$ \\
\hline Y101.1 & 9 & r r $\varepsilon . q$ & "ᄉ. & IV. & $0 . . \varepsilon$ & $7 \wedge V$ & NIV & المتوسط \\
\hline
\end{tabular}

المصدر : الاونكتار - تقرير الاستثمار في العالم، أعداد متفرقة. 


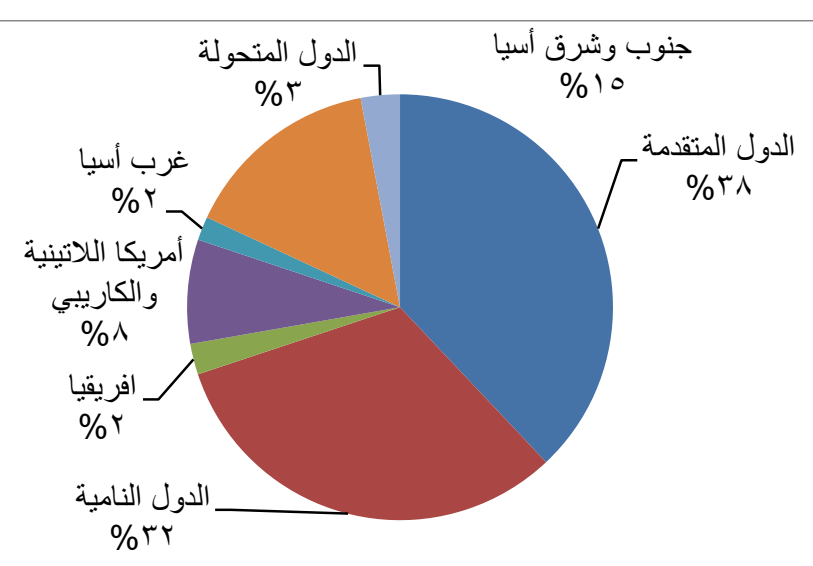

الشكل رقم (1) الأهمية النسبية للتدفقات العالمية للاستثمار الاجنبي المباشر الوارد خلال

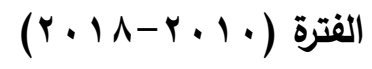

ثانيا: الوضع الحالي للأستثمار الأجنبي المباشر الصادرة من مناطق العالم: تشير بيانات الجدول رقم (Y) والثكل رقم (Y) الي حجم الاستثارات الأجنبية

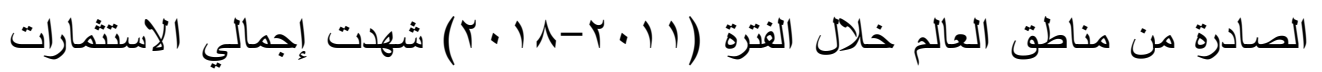
الأجنبية المباشرة الصادرة من مناطق العالم المختلفة تذبذبا واضحا خلال فترة الدراسة

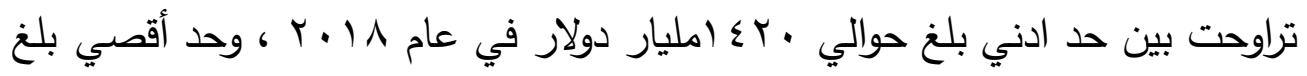

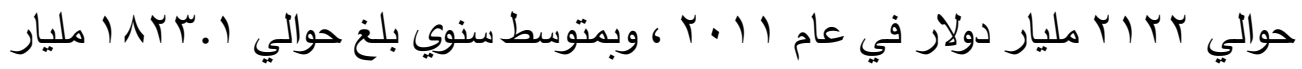
دولار ـ. أما حجم الاستثمارات الأجنبية الصادرة من الدول المتقدمة تراوحت بين حد ادني

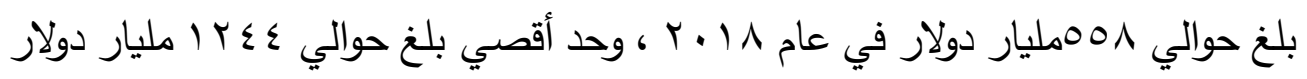

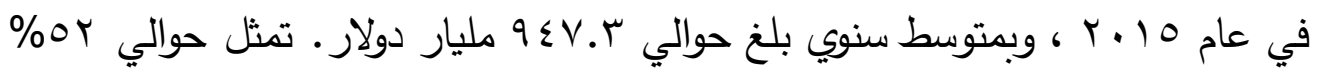
من جملة تلك الاستثمارات. أما حجم الاستثمارات الأجنبية الصادرة من الدول النامية

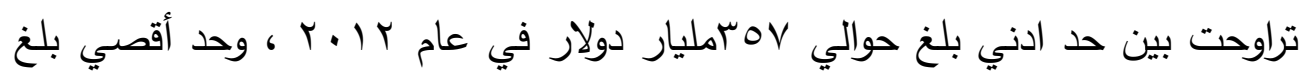

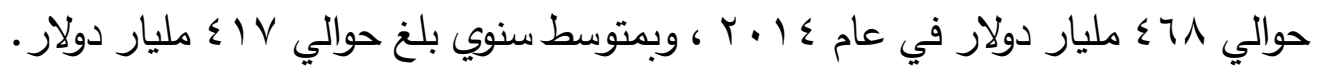


تمثل حوالي بr\% من جملة تلك الاستثمارات، أما حجم الاستثمارات الأجنبية الصادرة من افريقيا تراوحت بين حد ادني بلغ حوالي Vمليار دولار في عام II لـ ، وحد أقصي بلغ حوالي 17 مليار دولار في عام r| ـ ـ ، وبمتوسط سنوي بلغ حوالي ب. 11 مليار دولار • تمثل حوالي 0. • \% من جملة تلك الاستثمارات.

جدول رقم (Y) تطور التدفقات العالمية للاستثمار الاجنبي المباشر الصادرة خلال

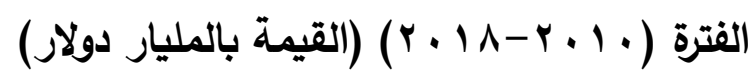

\begin{tabular}{|c|c|c|c|c|c|c|c|c|}
\hline الاجمالي & الدول & جنوب أسيا & غرب أسيا & أمريكا & افريقيا & النامية & الدولقدمة & السنوات \\
\hline rIrr & r & TV. & rr & 111 & V & ETr & $1 Y 17$ & 4.11 \\
\hline $17 r 9$ & $0 \leqslant$ & TצT & r & $\varepsilon \varepsilon$ & ir & rov & AVr & $r \cdot I r$ \\
\hline אודו & 91 & rqT & $\leqslant 1$ & rA & 17 & "N〉 & ATs & $r \cdot I r$ \\
\hline $1 \wedge 11$ & זד & rیr & H人 & rr & r & 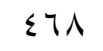 & ATr & $r \cdot 1 \varepsilon$ \\
\hline r. r & rt & TrE & $\leqslant 1$ & ro & 1. & $\varepsilon \cdot V$ & $\mid r \leq \varepsilon$ & $r .10$ \\
\hline 1971 & ro & Tor & $\leqslant 1$ & 11 & 9 & $\varepsilon r$. & 11.0 & $r .17$ \\
\hline IAVE & rq & דצ & $r q$ & צמ & rו & $\sum \pi r$ & gro & $r .1 T$ \\
\hline $1 \leqslant r$. & rᄉ & $\Gamma \leq$. & $\leqslant 9$ & $v$ & 1. & $\sum 1 \wedge$ & 001 & $r \cdot 1 \lambda$ \\
\hline | & 01.9 & ع.Trץ & ס.ק. & ro.7 & r. & 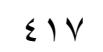 & $q \leq V . r$ & المتوسط \\
\hline
\end{tabular}

المصدر : الاونكتار - تقرير الاستثمار في العالم، أعداد متفرقة.

أما حجم الاستثمارات الأجنبية الصادرة من أمريكا اللاتينية تراوحت بين حد ادني

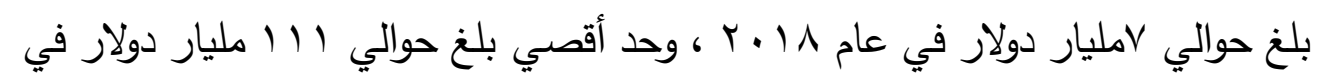

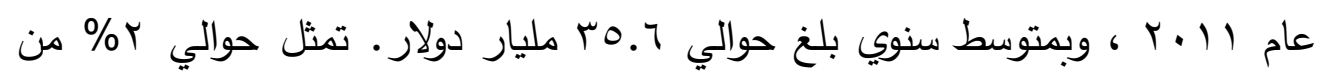
جملة تلك الاستثمارات، أما حجم الاستثمارات الأجنبية الصادرة من دول غرب أسيا 
تراوحت بين حد ادني بلغ حوالي ب rمليار دولار في عام II ـ ب ، وحد أقصي بلغ حوالي

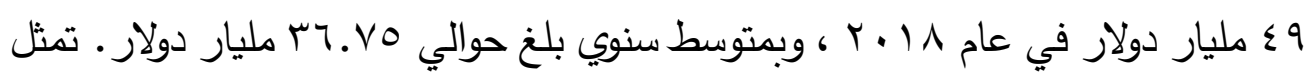
حوالي r\% من جملة تلك الاستثمارات، أما حجم الاستثمارات الأجنبية الصادرة من دول شرق وجنوب أسيا تراوحت بين حد ادني بلغ حوالي جابمليار دولار في عام

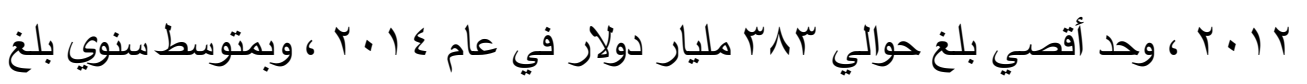
حوالي ع.بrr مليار دولار • تمثل حوالي ^| \% من جملة تلك الاستثمارات، أما حجم الاستثمارات الأجنبية الصادرة من الدول المتحولة تراوحت بين حد ادني بلغ حوالي

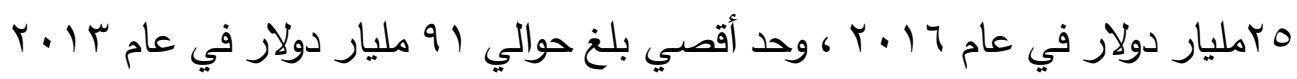
، وبمتوسط سنوي بلغ حوالي 9 . 0 مليار دولار • تمثل حوالي ب\% من جملة تلك

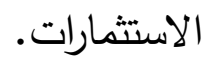

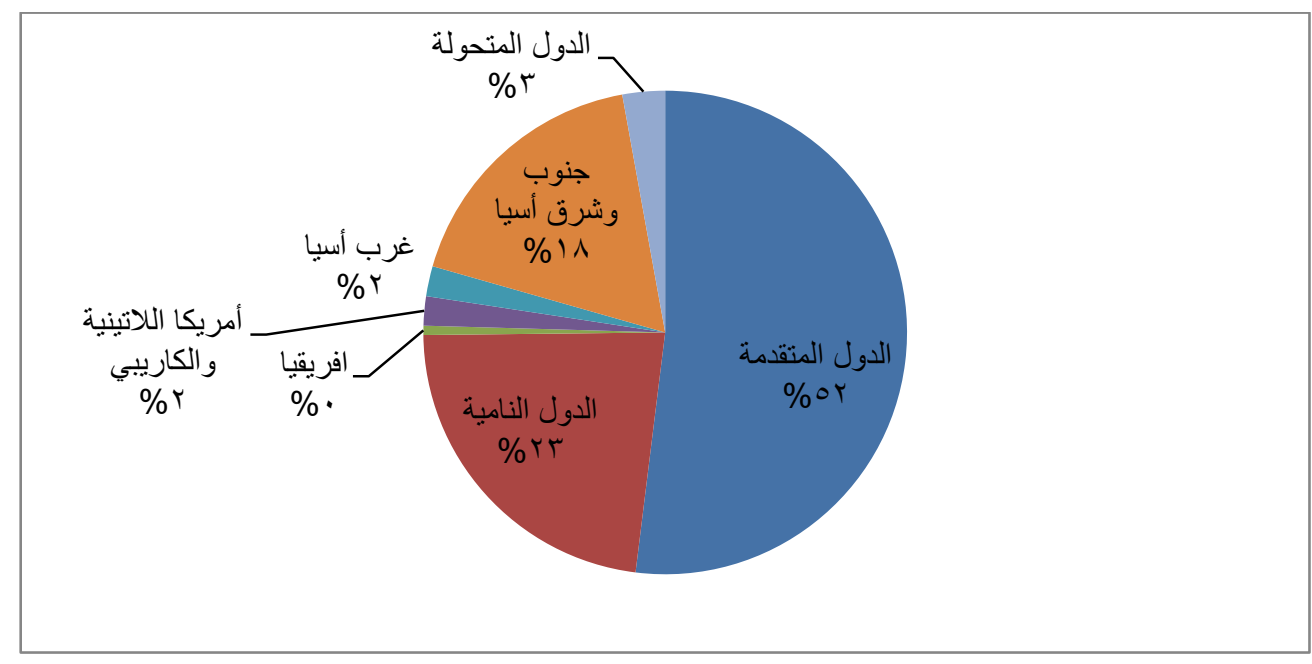

الثكل رقم (ץ) الأهمية النسبية للتدفقات العالمية للاستثمار الاجنبي المباشر الوارد

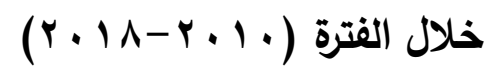


المطلب الثاني: التوزيع الجغرافي لنصيب دول الخليج العببية من الاستثمارات

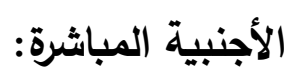

\section{اولأ: تطور تدفقات الاستثمارات الأجنبية المباشرة الواردة الي دول الخليج العببية:}

تشير بيانات الجدول رقم (r) والثكل رقم (r) الي حجم الاستثارات الأجنبية

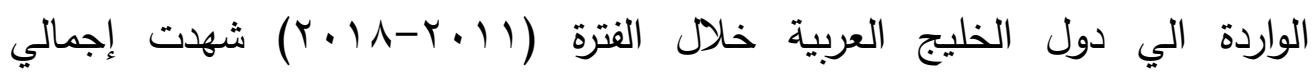
الاستثارات الأجنبية المباشرة الوارد الي دول الخليج العربية المختلفة تذبذبا واضحا

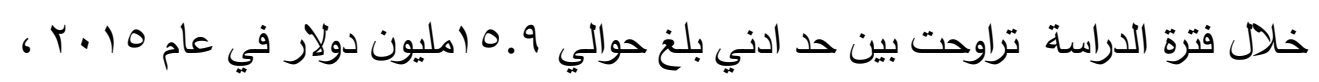

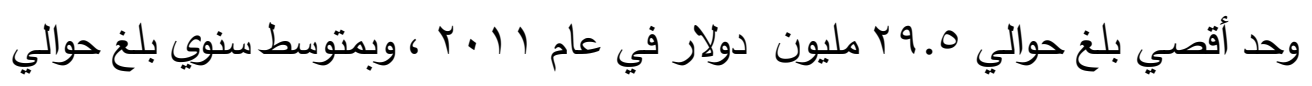

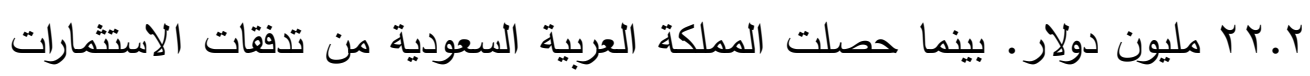

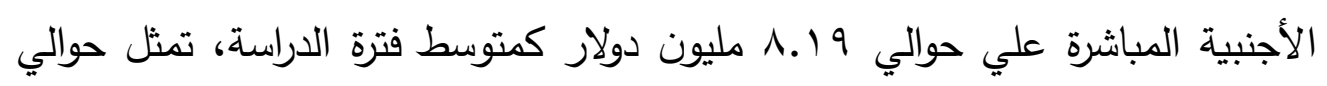

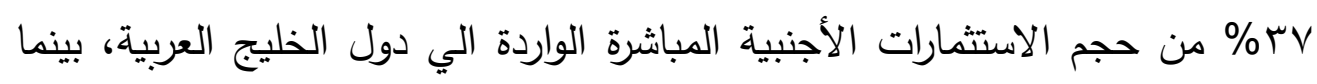

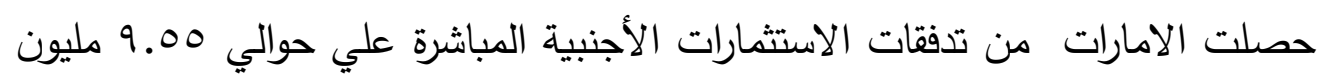

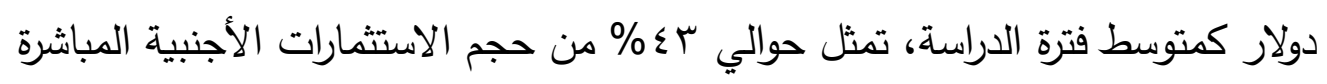
الواردة الي دول الخليج العربية، بينما حصلت دولة الكويت من تدفقات الاستثمارات

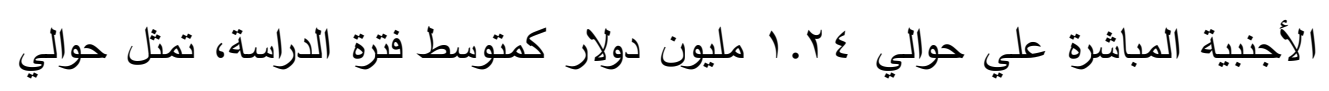

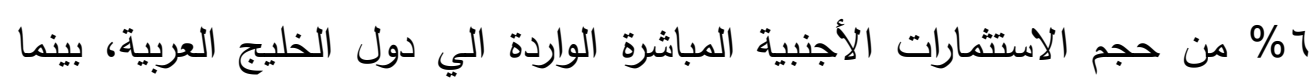

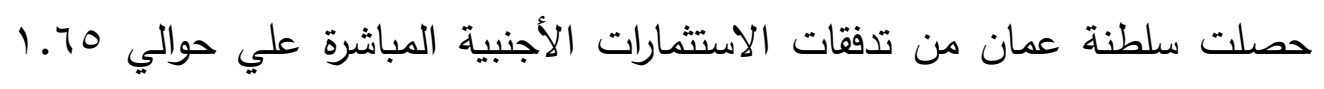

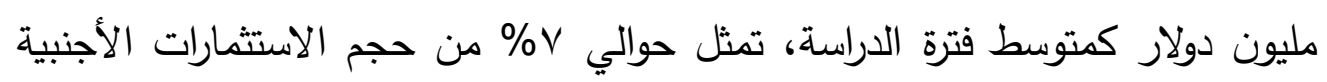

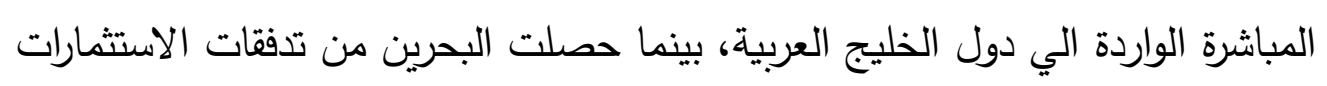

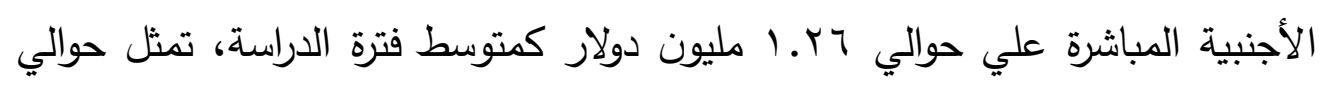
†\% من حجم الاستثمارات الأجنبية المباشرة الواردة الي دول الخليج العربية، بينما 
حصلت قطر من تدفقات الاستثمارات الأجنبية المباشرة علي حوالي rVY.. مليون دولار كمتوسط فترة الدراسة، تمثل حوالي ا \% من حجم الاستثمارات الأجنبية المباشرة الواردة الي دول الخليج العربية، وتحصلت الدول الثلاث السعودية والامارات والكويت علي ما يمثل ج^^\% من جملة الاستثمارات الأجنبية المباشرة الواردة الي دول الخليج العربية، أي أن باقي دول الخليج العربية تتحصل علي ؛ ا \% من هذه الاستثمارات.

جدول رقم (ץ) تطور تدفقات الاستثمارات الأجنبية المباشرة الواردة الي دول الخليج

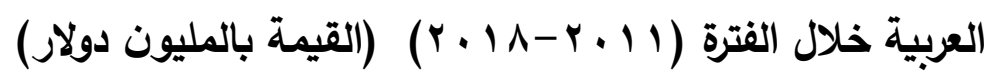

\begin{tabular}{|c|c|c|c|c|c|c|c|}
\hline الاجمالي & قطر & البحرين & سلطنة & الكويت & الامارات & السعودية & السنوات \\
\hline r9o.V & 9 qM & 91 & ivor & rroq & vior & $17 \pi \cdot 1$ & $r .11$ \\
\hline TVqYo & r9o & $10 \leqslant 0$ & 1570 & rAVT & 9077 & IrIAK & $r . I r$ \\
\hline$r \leqslant 07 r$ & $\Lambda \varepsilon \cdot-$ & TVYA & $171 \%$ & I & $9 \times 70$ & 1190 & $r \cdot 1 T$ \\
\hline rMAN & $1 . \varepsilon$. & 1011 & IYAV & 90\% & $11 . \times 1$ & $\lambda .1 Y$ & $r \cdot I \leq$ \\
\hline 10975 & $1 . v$. & T & r|V|- & r. & 100. & 人) $\leqslant$ & $r .10$ \\
\hline r.VOV & VVr & $r \leqslant r$ & TYTo & «ा & 97.0 & $V \leqslant O r$ & $r .17$ \\
\hline$|V \leq 0|$ & 917 & $1 \leq r 4$ & Yq1^ & $\Gamma \leqslant \wedge$ & 1. Tos & $1 \leqslant 19$ & $r . I V$ \\
\hline$I V \leqslant O V$ & $\begin{array}{c}- \\
\text { Y\人T }\end{array}$ & 1010 & $\varepsilon 19$ & $r \leqslant 0$ & $1 . r 10$ & r. & $r .11$ \\
\hline 22188 & 272 & 1267.1 & 1652.3 & 1242.3 & 9556 & 8198.5 & المتوسط \\
\hline
\end{tabular}

المصدر : الاونكتار - تقرير الاستثمار في العالم، أعداد متفرقة.

\section{اولأ: تطور تدفقات الاستثمارات الأجنبية المباشرة الصادرة من دول الخليج العربية:}

تثير بيانات الجدول رقم (ع) والثكل رقم (ع) الي حجم الاستثمارات الأجنبية الصادرة

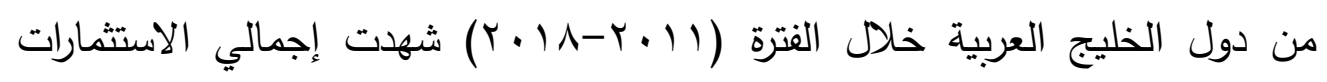


الأجنبية المباشرة الصادرة من دول الخليج العربية المختلفة تذبذبا واضحا خلال فترة

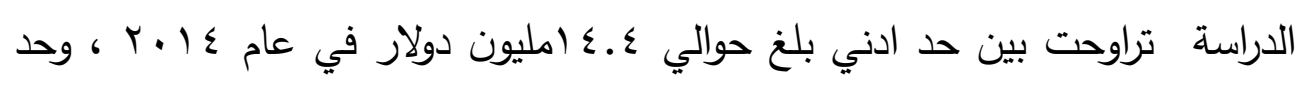

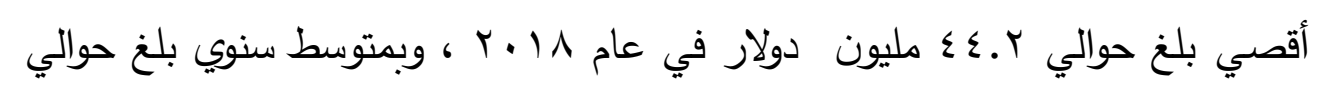

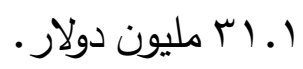

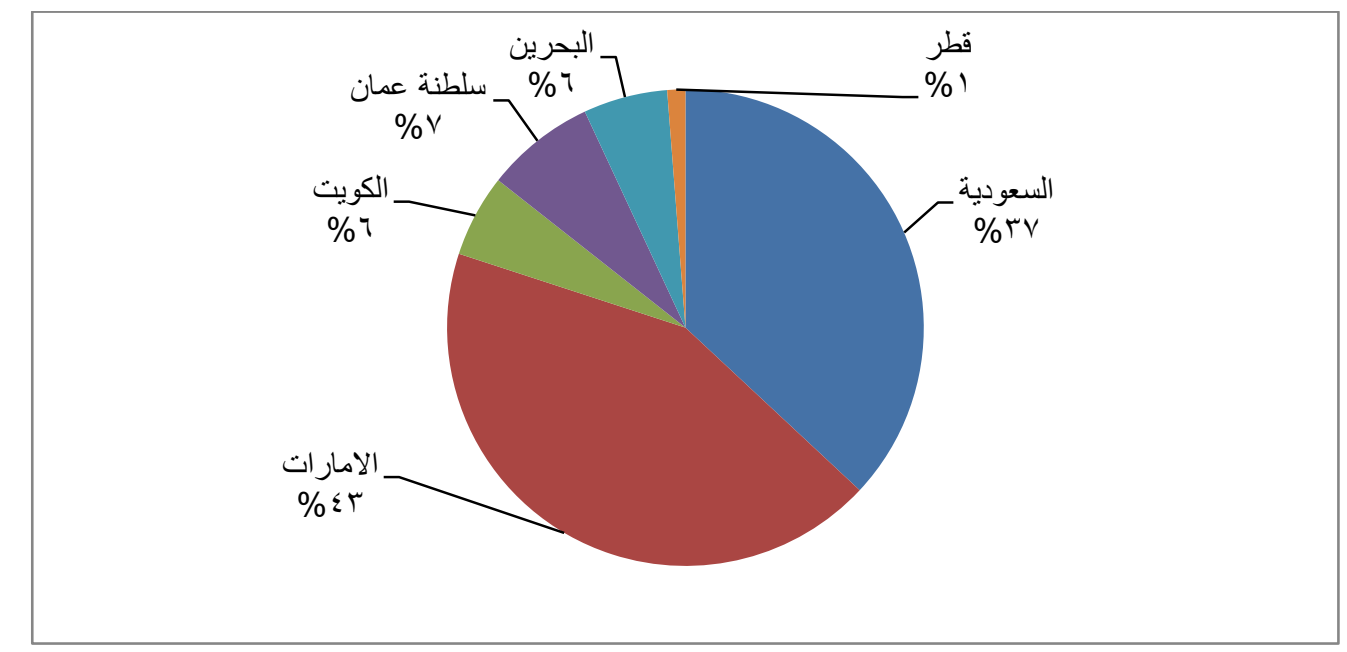

جدول رقم (r) الأهمية النسبية لتدفقات الاستثمارات الأجنبية المباشرة الواردة الي

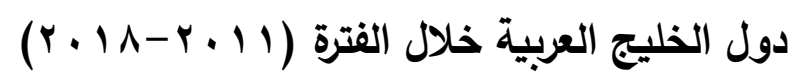

بينما بلغت حجم تدفقات الاستثمارات الأجنبية المباشرة الصادرة من المملكة

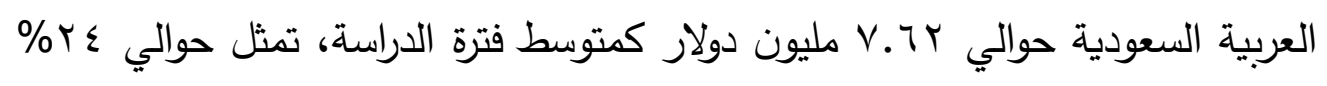
من حجم الاستثمارات الأجنبية المباشرة الصادرة من دول الخليج العربية، بينما بلغت حجم تدفقات الاستثمارات الأجنبية المباشرة الصادرة من الامارات حوالي 9. دولار كمتوسط فترة الدراسة، تمثل حوالي هب\% من حجم الاستثمارات الأجنبية المباشرة الصادرة من دول الخليج العربية، بينما بلغت حجم تدفقات الاستثمارات الأجنبية المباشرة الصادرة من دول الكويت حوالي ^.0 مليون دولار كمتوسط فترة الدراسة، تمثل حوالي 9 1\% من حجم الاستثمارات الأجنبية المباشرة الصادرة من دول الخليج العربية، بينما بلغت حجم تدفقات الاستثمارات الأجنبية المباشرة الصادرة من سلطنة عمان 
حوالي 1..1 مليون دولار كمتوسط فترة الدراسة، تمثل حوالي ب\% من حجم الاستثمارات الأجنبية المباشرة الصادرة من دول الخليج العربية، بينما بلغت حجم تدفقات الاستثمارات الأجنبية المباشرة الصادرة من البحرين حوالي ^ه؟. . مليون دولار كمتوسط فترة الدراسة، تمثل حوالي ا ٪ من حجم الاستثمارات الأجنبية المباشرة الصادرة من دول الخليج العربية، بينما بلغت حجم تدفقات الاستثمارات الأجنبية المباشرة الصادرة من قطر حوالي 0.0 مليون دولار كمتوسط فترة الدراسة، تمثل حوالي 11\% من حجم الاستثمارات الأجنبية المباشرة الصادرة من دول الخليج العربية، واحتلت الدول الثلاث السعودية والامارات والكويت علي ما يمثل ٪^\% من جملة الاستثمارات الأجنبية المباشرة الصادرة من دول الخليج العربية، أي أن باقي دول الخليج العربية تمثل حوالي r r\% من هذه الاستثمارات.

دول رقم (؛) تطور تدفقات الاستثمارات الأجنبية المباشرة الصادرة من دول الخليج

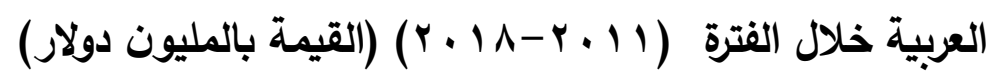

\begin{tabular}{|c|c|c|c|c|c|c|c|}
\hline الاجمالي & قطر & البحرين & سلطنة & الكويت & الامارات & السعودية & السنوات \\
\hline rqva. & $1 \cdot 1 \cdot 1$ & qr.- & ITYK & $1 \cdot V V Y$ & YIVA & $\Gamma \leqslant \mu$. & $r .11$ \\
\hline 17919 & $1 \wedge \varepsilon$. & 017 & $\wedge \wedge \varepsilon$ & $T \vee \leqslant 1$ & rory & $\varepsilon \varepsilon \cdot r$ & $r .1 T$ \\
\hline एqq. & $A \cdot Y_{1}$ & OrY & rm & $177 \leqslant \wedge$ & 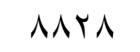 & $\varepsilon q \leq r$ & $r .1 T$ \\
\hline $1 \leqslant$ rVo & $7 \vee \leqslant \Lambda$ & rqr- & ITOV & $1 \cdot \leqslant 7 \wedge-$ & I I I & orq7 & $r .1 \varepsilon$ \\
\hline$r \leqslant 99 \wedge$ & r. & 191 & דאץ & ortV & 17791 & orq. & $r .10$ \\
\hline r.001 & $\vee q .1$ & $\wedge \wedge \cdot-$ & rot & sorv & $10 \times 11$ & רצr & $r .17$ \\
\hline$r \leqslant \vee \ldots$ & 1790 & $r r q$ & $r \leq r \leq$ & q. & $1 \varepsilon .09$ & $\checkmark Y \wedge$. & $r . I V$ \\
\hline$\varepsilon \leqslant Y \leqslant \Lambda$ & TOYT & 111 & 077 & rvol & 10.19 & $r|r| q$ & $r .11$ \\
\hline 31061 & 5482.3 & 298.25 & 1009.8 & 5793.9 & 10852 & 7624.5 & لمتوسط \\
\hline
\end{tabular}

المصدر : الاونكتار - تقرير الاستثمار في العالم، أعداد متفرقة. 
المطلب الثالث: تطور مشاريع الاستثمار الأجنبي المباشر الجديدة الواردة الي دول الخليج العربية:

اولأ: تطور مشاريع الاستثمار الأجنبي العباشر لاول الخليج العببية خلال الفترة من $:(r \cdot 1 \Lambda-r \cdot 11)$

تثير بيانات جدول رقم (0) والثكل رقم (0) الي تطور مشاريع الاستثمار

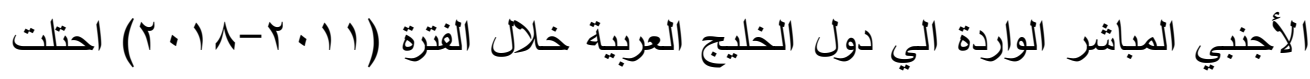

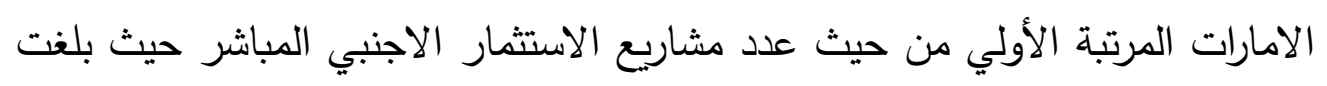

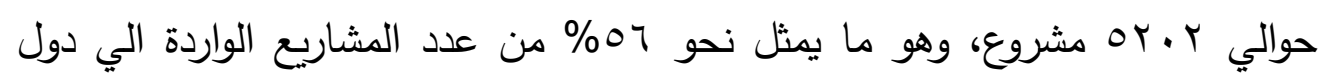

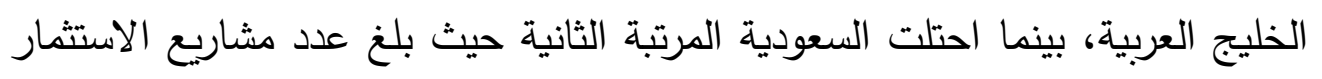

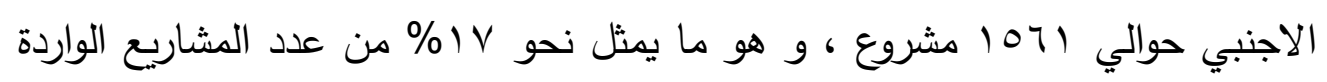

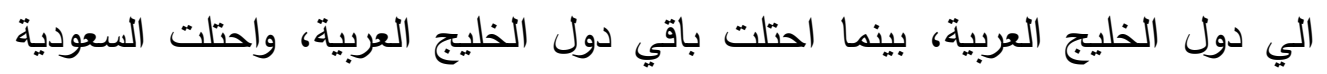

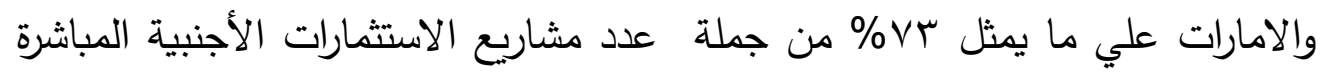

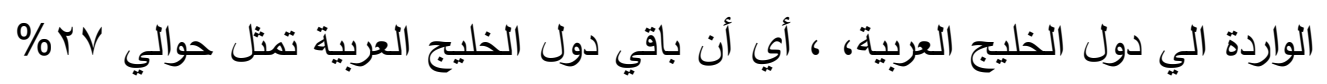
من هذه الاستثمارات.

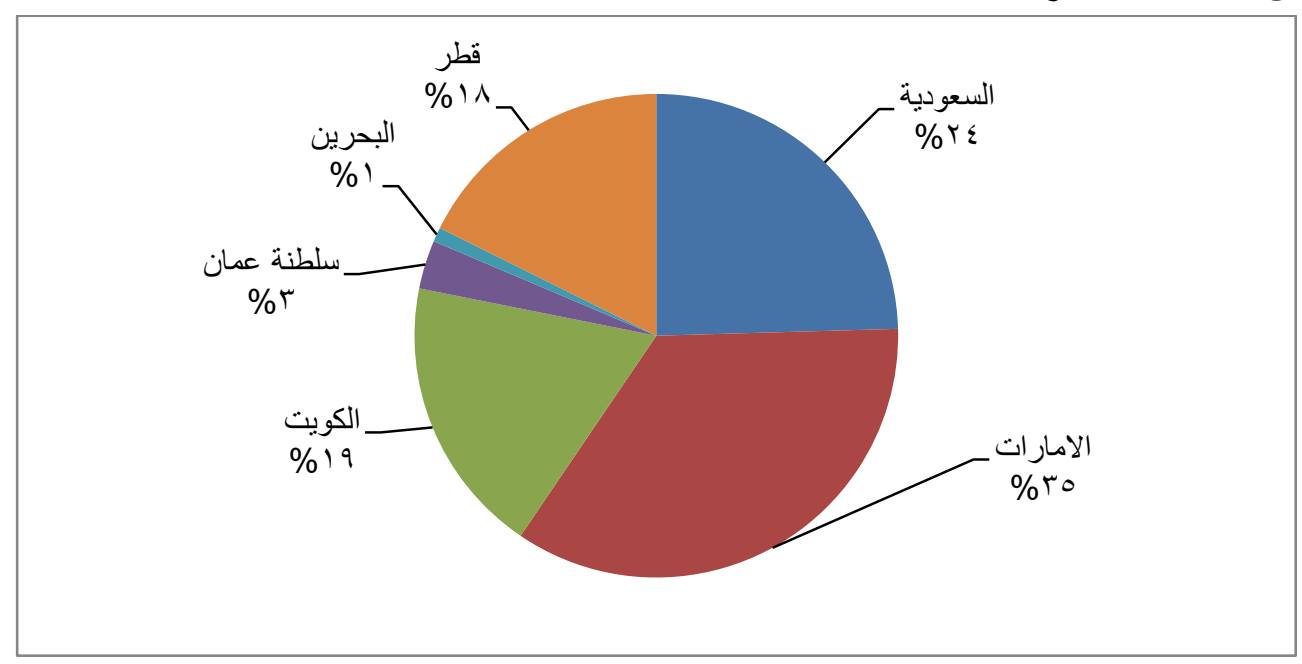

جدول رقم (ع) الأهمية النسبية لتدفقات الاستثمارات الأجنبية المباشرة الصادرة من

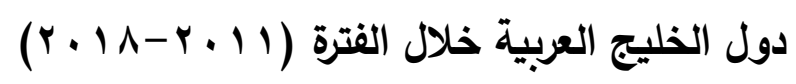


الجدول رقم (0) تطور مشاربع الاستثمار الأجنبي المباشر الجديدة الواردة الي دول

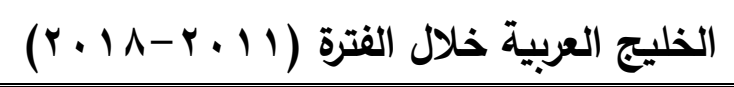

\begin{tabular}{|c|c|c|c|c|}
\hline عدد الشركات & عدد الوظائف & 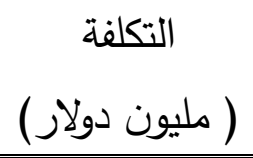 & عدد المشاريع & الدولة \\
\hline $11 \cdot \varepsilon$ & $I V E \Lambda \mu$. & $\mid \vee q \vee 1 \leq$ & 1071 & السعودية \\
\hline$\leq r \leq 0$ & $\leq r \leq T)$. & $1 v 1194$ & Or. r & الامارات \\
\hline$r \cdot \Lambda$ & 1 & | & rql & الكويت \\
\hline$\varepsilon V$. & $1 \cdot 1 \leqslant 97$ & VOITY & 770 & سلطنة عمان \\
\hline 077 & $\wedge 9 \leq \leq 1$ & 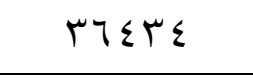 & $V \cdot T$ & البحرين \\
\hline 795 & $9 \longleftrightarrow \wedge \leq V$ & $910 Y \varepsilon$ & Nז人 & قطر \\
\hline
\end{tabular}

المصدر : الاونكتار - تقرير الاستثمار في العالم، أعداد متفرقة.

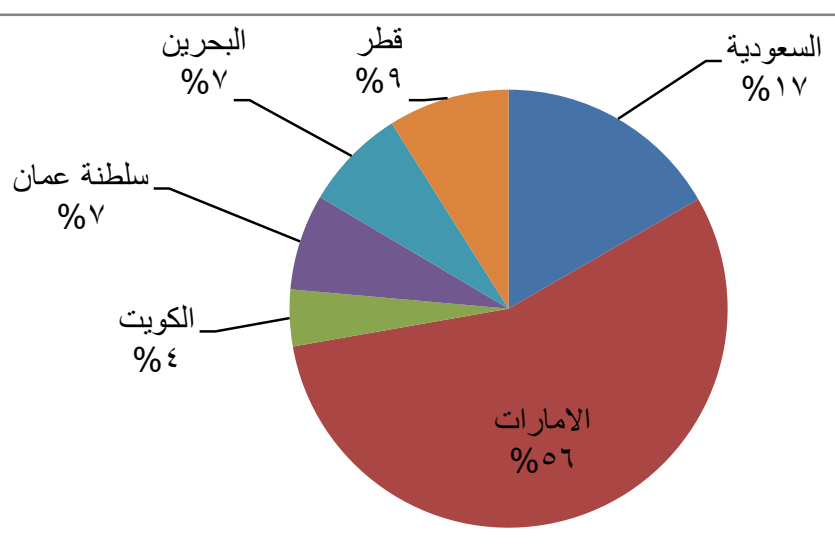

الثكل رقم (0) الاهمية النسبية لعدد مشاريع الاستثمار الأجنبي المباشر الجديدة

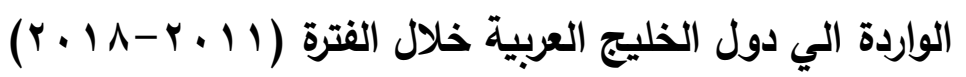


ثانيا: التوزيع الجغرافي للاستثمارات البينية لاول الخليج العربية خلال الفترة من : r.1N-r.1·)

تثير بيانات جدول رقم(T) الي مصفوفة الاستثمارات البينية لدول الخليج العربية

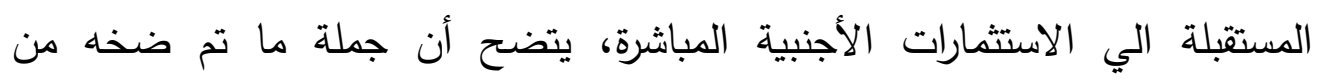

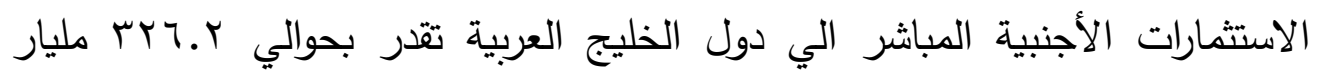

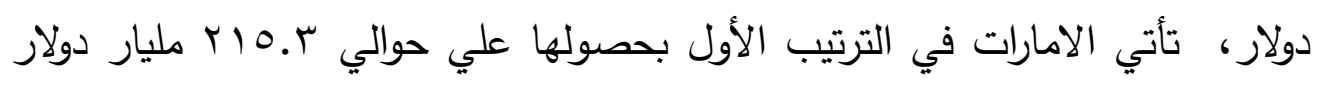

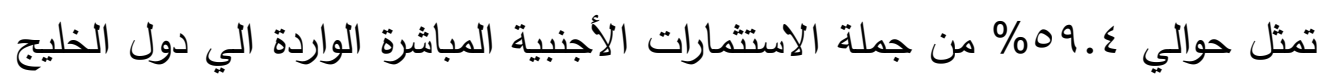

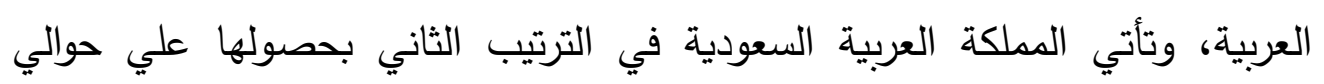

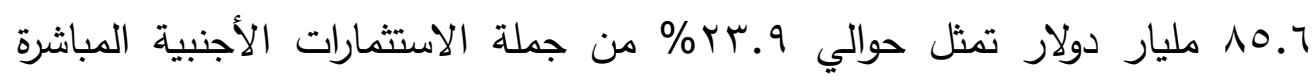

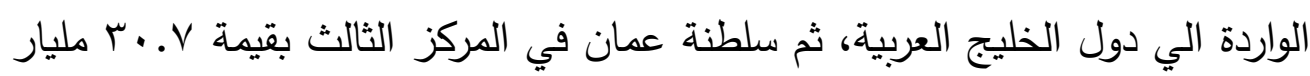

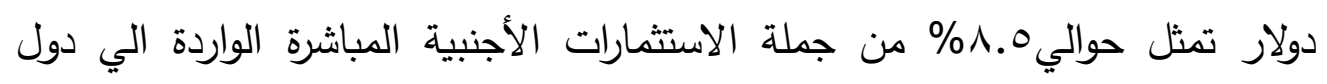

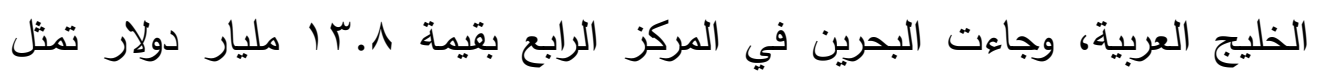

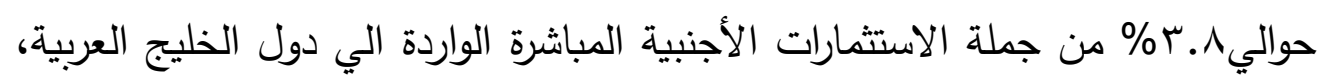

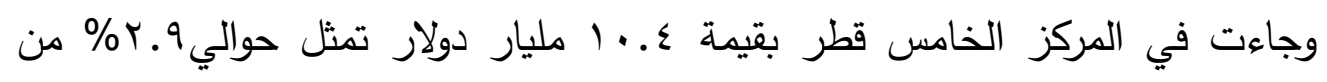

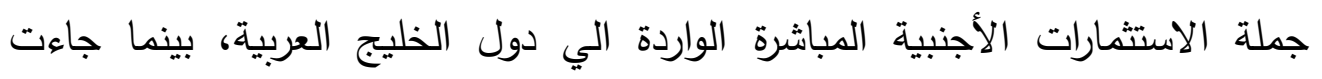

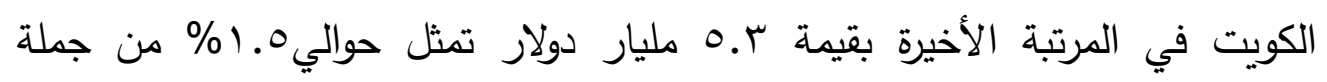
الاستثارات الأجنبية المباشرة الواردة الي دول الخليجة العية العربية. بينما تثير بيانات نفس الجدول السابق الي مصفوفة الاستثمارات البينية

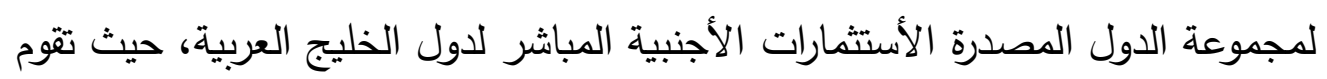

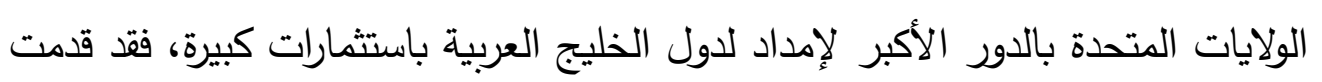

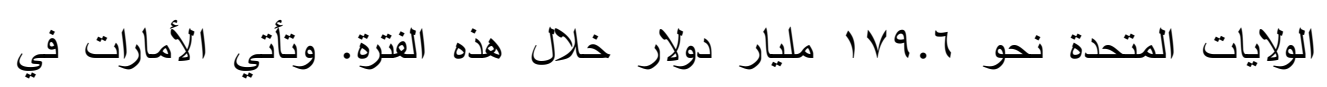

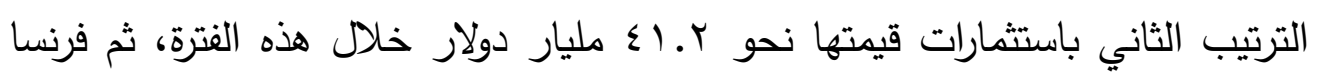

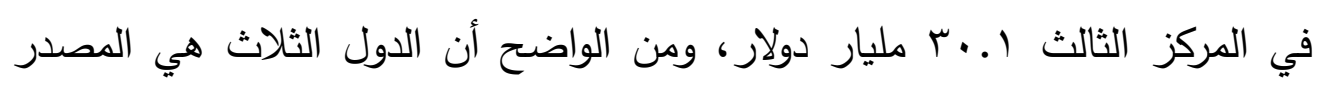
الأساسي للاستثمارات الجنبية المباشرة لدول الخليج العربية. 


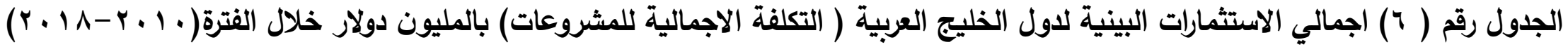

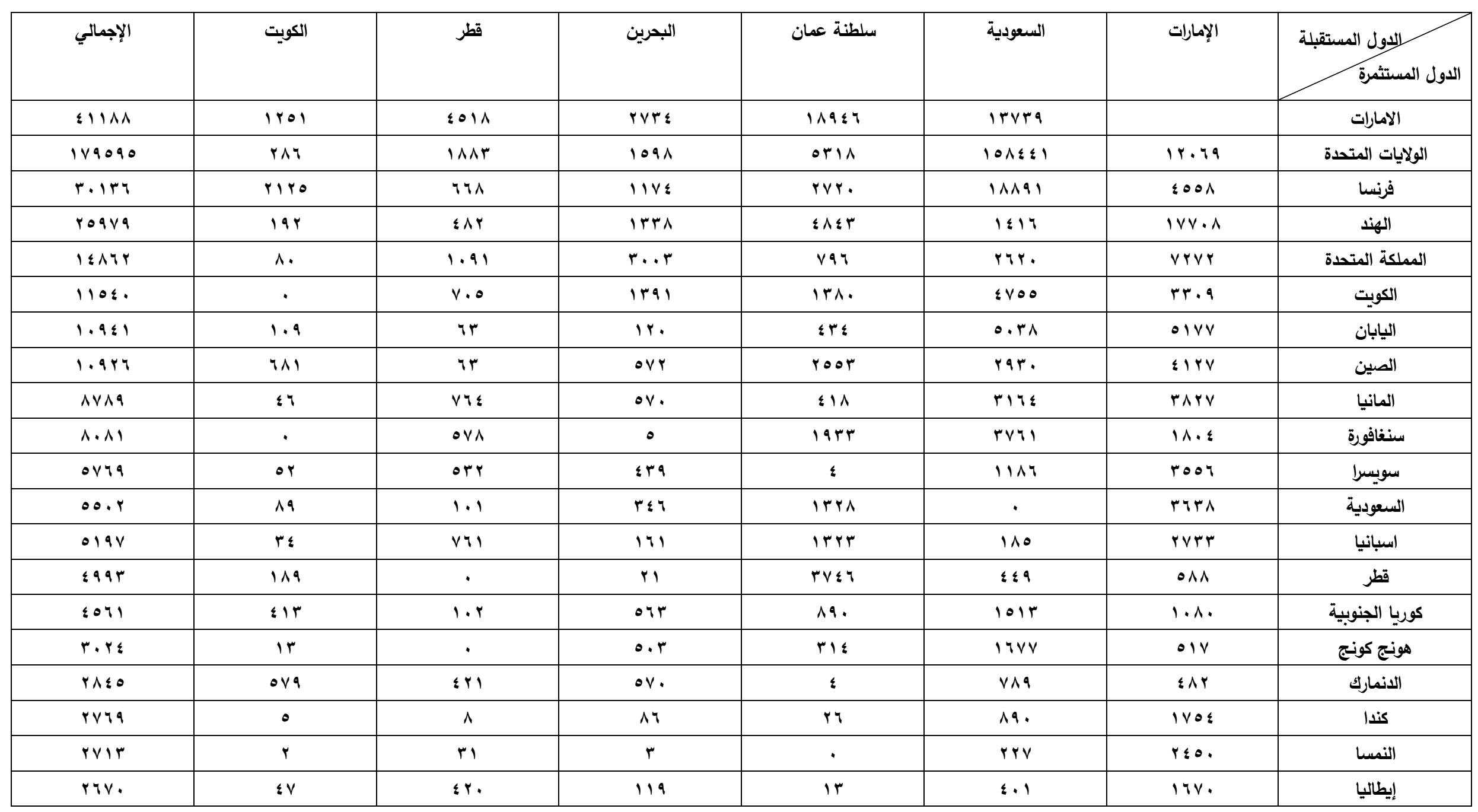




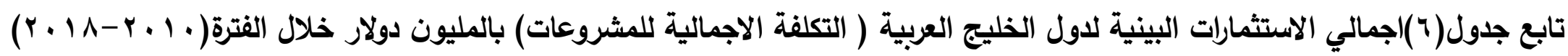

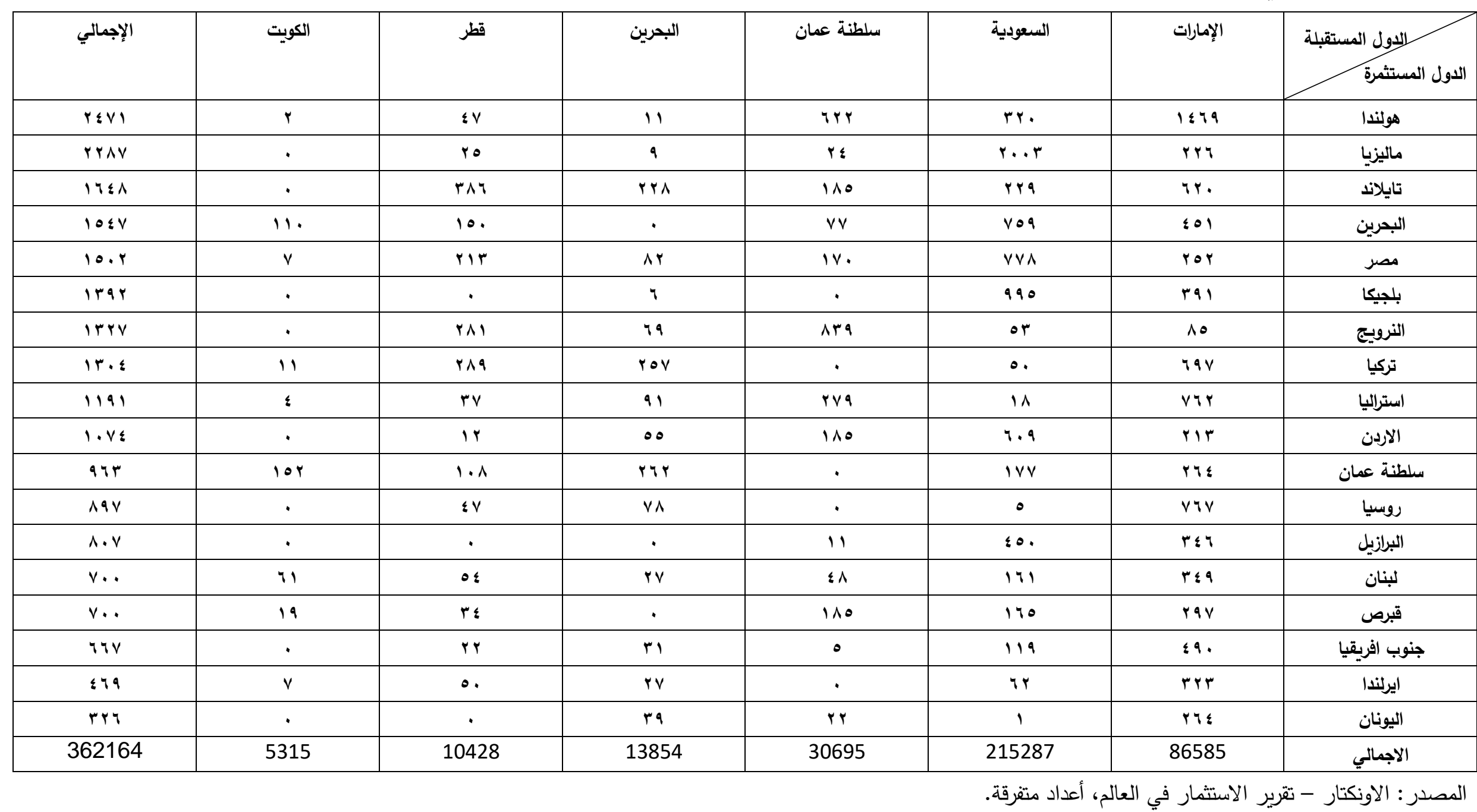




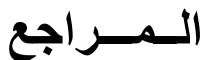

1- إمام محمود الجمسي، الجوانب الاقتصادية للاستثمارات الأجنبية المباشرة في الدول العربية ونصيب الزراعة منها، مؤتمر الاستثمار والتتمية في الوطن العربي، الاتحاد العربي

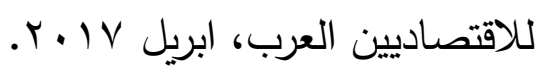

r-بلال لوعيل، تطور الاستثمارات الجنبية المباشرة العببية البينية، كلية العلوم الاقتصادية والتجارة وعلوم التسيير، جامعة محمد بوفرة، الجزائر، 10 ب r. r- البنك الدولي، قاعدة بيانات احصاءات التتمية الاقتصادية. ع- ححم اسماعيل ، جمال قاسم ، محددات الاستثمار الأجنبي المباشر في الدول العربية،

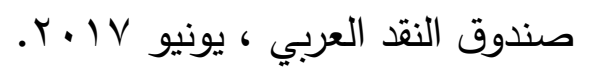

